\title{
Desempenho produtivo de vacas recebendo dietas com capim-elefante submetido a diferentes adubações e níveis de concentrado
}

\author{
Tadeu Silva de Oliveira ${ }^{1}$, José Carlos Pereira², Augusto César de Queiroz ${ }^{2}$, Paulo Roberto Cecon ${ }^{3}$, \\ Márcia Vitória dos Santos ${ }^{1}$
}

\begin{abstract}
RESUMO
Os objetivos deste trabalho foram determinar o consumo de matéria seca total (MSDT), a produção e composição do leite de vacas lactantes quando submetidas a dietas à base de capim-elefante adubado quimicamente (CEAQ) ou organicamente (CEAO) e a energia líquida para lactação (ELL) e os nutrientes digestíveis totais (NDT) do capimelefante na dieta. O CEAQ ou CEAO foi misturado ao concentrado em níveis de 400, 500 e $600 \mathrm{~g}$ na base da matéria seca (MS). Os tratamentos foram dispostos em delineamento em quadrado latino 6 x 6, esquema fatorial de 2 x 3 (adubação $\mathrm{x}$ nível de concentrado). Os teores médios de MSDT e os de FDN, PB, Ca e P da dieta na MS diferiram $(\mathrm{P}<0,05)$ para os diferentes níveis de concentrado. O consumo da MSDT e dos nutrientes aumentou $(\mathrm{P}<0,05)$ com a elevação dos níveis de concentrado, exceto para FDN. O capim adubado organicamente diminuiu o consumo da MSDT e da FDN da dieta total. A digestão da MS, MO e PB da dieta total não sofreu influência dos níveis de concentrado e foi para o CEAO. Houve queda $(\mathrm{P}<0,05)$ na digestão da FDN da dieta com o aumento nos níveis de concentrado. A produção e composição do leite não variaram com os níveis de concentrado nem com as diferentes adubações. O valor energético (ELL e NDT) do capim-elefante decresceu $(\mathrm{P}<0,05)$ à medida que os níveis de concentrado aumentaram na dieta e não foi influenciado pelo sistema de adubação. A eficiência de síntese de proteína microbiana em relação à PB da dieta consumida foi melhor para o nível de $400 \mathrm{~g}$ de concentrado.
\end{abstract}

Palavras-chave: Consumo, performance produtiva, vacas mestiças.

\section{ABSTRACT \\ Yield performance of cows fed diets with elephant grass subjected to different fertilization and concentrate levels}

The objectives of this work were to determine the total diet dry matter (TDDM) intake, milk production and milk composition, net energy for lactation (TNE) and total digestible nutrients (TDN) of elephant grass in the total diet. The elephant grass subjected to chemical (CF) and organic (OF) fertilization was mixed to the concentrate, at levels of 400, 500 and $600 \mathrm{~g}$, in dry matter basis (DM). The treatments were arranged in a 6 x 6 Latin square, in a 2 x 3 factorial design (fertilizer $\mathrm{x}$ concentrate level). The average TDDM contents, in fed basis, and NDF, CP, Ca and P of the total diet, in DM basis, were different $(\mathrm{P}<.05)$ for the different concentrate levels. The TDDM and nutrient intakes increased $(\mathrm{P}<.05)$ with increasing concentrate levels, except for NDF $(\mathrm{P}>0,05)$. Concentrate levels did not influence digestion of TDDM, $\mathrm{MO}$ and $\mathrm{PB}$ of total diet. NDF digestion decreased $(\mathrm{P}<.05)$ with increasing concentrate levels. Digestion of DM, OM

\footnotetext{
Recebido para publicação em 10/12/2010 e aprovado em 13/04/2011

${ }^{1}$ Zootecnista, Mestre. Departamento de Zootecnia, Universidade Federal de Viçosa, Av. Peter Henry Rolfs, s/n, 36571-000, Viçosa, Minas Gerais, Brasil. tadeusilva@zootecnista.com.br; marciavitori@hotmail.com

${ }^{2}$ Engenheiro-Agrônomo, Doutor. Departamento de Zootecnia, Universidade Federal de Viçosa, Av. Peter Henry Rolfs, s/n, 36571-000, Viçosa, Minas Gerais, Brasil. jcarlos@ ufv.br; aqueriroz@ufv.br

${ }^{3}$ Engenheiro-Agrônomo, Doutor. Departamento de Estatística, Universidade Federal de Viçosa, Av. Peter Henry Rolfs, s/n, 36571-000, Viçosa, Minas Gerais, Brasil. pcecon@ ufv.br
} 
and $\mathrm{CP}$, and of FDN of total diet with elephant grass subjected to OF were higher than total diet with elephant grass subjected to CF. Milk production and milk composition did not change with increasing concentrate levels. The energy value (ELL and NDT) of elephant grass decreased $(\mathrm{P}<.05)$ an average of $15 \%$ with increasing concentrate levels and did not change with the different fertilizers. The efficiency of microbial protein synthesis in relation to the $\mathrm{CP}$ in the total diet consumed was best at $400 \mathrm{~g}$ of concentrate, showing the lowest feed: gain ratio.

Key words: Crossbred cows, intake, yield performance.

\section{INTRODUÇÃO}

No custo de produção de leite, a alimentação é o componente mais significativo dos custos variáveis, e os alimentos concentrados são os de maior custo na alimentação; ou seja, é o componente de maior relevância no custo de produção do leite. Desse modo, é importante a otimização da relação entre alimentos volumosos e concentrados fornecidos aos animais, uma vez que quanto maior a quantidade de volumosos em relação aos concentrados menor o custo com alimentação (Vilela et al., 1996).

Um dos aspectos básicos na atividade leiteira é procurar obter a máxima eficiência de utilização dos nutrientes da ração. $\mathrm{O}$ ingrediente volumoso desempenha um papel insubstituível, porque, além de sua função nutricional, desencadeia e realça os processos fisiológicos de ruminação e mastigação, mantendo saudável o ecossistema ruminal, para desempenhar com eficácia a atividade fermentativa.

A manipulação da dieta, com o intuito de alterar a produção e a composição do leite, vem-se tornando muito comum dentro da atividade leiteira, sendo a produção de leite e o teor de gordura os mais influenciados pela dieta (Oliveira et al., 2007).

O suprimento energético e os precursores da gordura e dos carboidratos do leite são oriundos, principalmente, do processo de fermentação, que acontece no rúmenretículo e ceco (Van Soest, 1994). O consumo de matéria seca (CMS) é fator limitante na produção de leite, cujo controle pode ser feito em três níveis: comportamental, fisiológico e físico (Mertens, 1994).

A relação volumoso:concentrado é importante, pois a sua queda causa incremento na quantidade de carboidratos fermentáveis no rúmen, que provocam redução no $\mathrm{pH}$ ruminal mediante o aumento dos ácidos orgânicos produzidos (Oliveira et al., 2007).

Wattiaux (1994) relatou o efeito da relação volumoso: concentrado (V:C) na proporção de ácido acético e ácido propiônico e no $\mathrm{pH}$ do rúmen, também verificou esse efeito na produção e no teor de gordura do leite, observando que ao se variar a relação $\mathrm{V}: \mathrm{C}$ em uma amplitude de 80:20 a 20:80 a relação de ácidos acético e propiônico decaiu na proporção de 6,5:1 a 1:1; o pH, de 7,0 para 5,25; a produção de leite se elevou até a relação 30:70; e o teor de gordura do leite permaneceu estável até a relação 40:60, decaindo em relação abaixo dessa.

O equilíbrio no ambiente ruminal é um ponto essencial na nutrição de vacas em lactação. Portanto, deve-se buscar equilíbrio na ração para se obter a máxima eficiência possível.

Os objetivos deste trabalho foram determinar o consumo de matéria seca (CMS) e a quantidade de nutrientes degradados no rúmen e avaliar a produção e a composição química do leite, bem como estimar a energia líquida para lactação (ELL) e os nutrientes digestíveis totais (NDT) do capim-elefante na dieta total.

\section{MATERIAL E MÉTODOS}

O experimento foi realizado nas dependências do Departamento de Zootecnia da Universidade Federal de Viçosa (UFV), situada na cidade de Viçosa, Estado de Minas Gerais, Zona da Mata Atlântica, a $20^{\circ} 45^{\prime}$ de latitude sul e $42^{\circ} 51^{\prime}$ de longitude oeste, à altitude de $649 \mathrm{~m}$. O clima de Viçosa é subtropical, com inverno frio e seco e verão quente e úmido.

Foram utilizadas seis vacas mestiças com grau genético entre 5/8 e 31/32 Holandês-Zebu, pesando, em média, $504 \mathrm{~kg}(\mathrm{CV}=15 \%)$, com 40 a 57 dias de lactação e produzindo, em média, $18,50 \mathrm{~kg}(\mathrm{CV}=17,14 \%)$ de leite com $4 \%$ de gordura.

Foi implementado um delineamento em quadrado latino em esquema fatorial $2 \times 3$ : capim-elefante adubado de formas orgânica (CEO) e química (CEQ), associado ao concentrado em três níveis, $40 ; 50$ e $60 \%$, com base na matéria seca (MS).

As dietas experimentais foram ajustadas semanalmente, em função da MS do capim-elefante e das sobras, que variaram de 5 a 10\%. Constam na Tabela 1 os ingredientes e a composição químico-bromatológica do concentrado, já na Tabela 2 composição químico-bromatológica do capim-elefante. 
O experimento teve duração de 126 dias, durante o qual os animais foram mantidos em regime de confinamento do tipo tie-stall alimentados às $7 \mathrm{~h}$ e $15 \mathrm{~h} 30, \log$ após as ordenhas, que ocorriam as 6 e 15 h. Às 19 h, eram soltas em área de descanso com piso de terra.

A duração de cada período experimental foi de 21 dias, sendo sete para adaptação dos animais e 14 de coleta de dados, sendo os animais pesados no último dia do período de adaptação e no último dia do período de coletas. As pesagens das vacas foram feitas sem jejum prévio após a ordenha da manhã.

Amostras da dieta total e das sobras, correspondentes a cada vaca, foram coletadas duas vezes ao dia, durante todo o período, e as amostras dos volumosos, coletadas duas vezes ao dia no $1^{\circ}, 4^{\circ}, 7^{\circ}, 10^{\circ}$ e $13^{\circ} \stackrel{\circ}{\text { dias. }}$

As amostras de fezes foram coletadas nos segundo, quarto e sexto períodos, do $7^{\circ}$ ao $14^{\circ}$ dia, antes da alimentação da manhã e da tarde, diretamente no reto de cada animal.

O leite foi amostrado proporcionalmente à produção de cada ordenha, duas vezes por semana, durante o período de coletas, sendo as amostras mantidas em refrigeração a 4 ${ }^{\circ} \mathrm{C}$, para análises de densidade e gordura, e outra alíquota foi utilizada para posterior determinação da composição.

Tabela 1. Proporção dos ingredientes e composição químicobromatológica do concentrado

\begin{tabular}{|c|c|}
\hline Ingredientes & $\% \mathrm{MS}$ \\
\hline Fubá de milho & 72,42 \\
\hline Farelo de soja & 25,34 \\
\hline Cloreto de sódio & 0,51 \\
\hline Fosfato bicálcico & 0,95 \\
\hline Calcário calcítico & 0,70 \\
\hline Premix mineral ${ }^{1}$ & 0,02 \\
\hline Premix vitamínico ${ }^{2}$ & 0,06 \\
\hline \multicolumn{2}{|l|}{ Composição químico-bromatológica } \\
\hline Proteína bruta $(\mathrm{PB})$ & 19,80 \\
\hline Fibra em detergente neutro (FDN)) & 10,15 \\
\hline $\mathrm{Ca}$ & 0,61 \\
\hline $\mathrm{P}$ & 0,47 \\
\hline $\mathrm{Na}$ & 0,39 \\
\hline $\mathrm{K}$ & 0,92 \\
\hline
\end{tabular}

Incluídos 113,50 g de $\mathrm{Zn} / \mathrm{kg}, 106,25 \mathrm{~g}$ de $\mathrm{Cu} / \mathrm{kg}, 112,37 \mathrm{~g}$ de $\mathrm{S} / \mathrm{kg}$, $21,11 \mathrm{~g}$ de I/kg, 12,69 g de Se/kg, 7,69 g de Na/kg, 6,89 g de Co/kg e $6,67 \mathrm{~g}$ de $\mathrm{K} / \mathrm{kg} .{ }^{2}$ Incluídos $10.500 \mathrm{UI}$ de vitamina A/g, 2.500 UI de vitamina $\mathrm{D}_{3} / \mathrm{g}$ e 15 UI de vitamina $\mathrm{E} / \mathrm{g}$
Além dessas amostragens, a cada sete dias foram coletadas amostras dos volumosos para determinação imediata de MS e ajuste da relação volumoso:concentrado da dieta total.

Os teores de matéria seca (MS), matéria orgânica (MO), proteína bruta $(\mathrm{PB})$, cálcio $(\mathrm{Ca})$, fósforo $(\mathrm{P})$, magnésio $(\mathrm{Mg})$, sódio $(\mathrm{Na})$ e potássio $(\mathrm{K})$ foram determinados conforme métodos descritos por Silva e Queiroz (2002), e a fibra em detergente neutro (FDN) foi determinada conforme Van Soest et al. (1991).

Os teores de gordura das amostras de leite foram verificados conforme método de Gerber (Coelho \& Rocha, 1981), e a densidade, por intermédio do termolactodensímetro, calibrado a $15^{\circ} \mathrm{C}$ e corrigido para a temperatura da amostra. Os sólidos totais do leite (STL) foram determinados a partir da seguinte fórmula citada por Mora (1995): $S T L=\{1,2+g+2,665 \times[(100 x d)-100]\} / d$, em que $\mathrm{STL}=$ sólidos totais do leite $(\%) ; \mathrm{g}=$ teor de gordura do leite $(\%)$; e $\mathrm{d}=$ densidade do leite $\left(\mathrm{g} / \mathrm{cm}^{3}\right)$.

A pesagem do leite de cada vaca foi feita em dias alternados durante o período de coleta. A produção média (kg de leite $\mathrm{vaca}^{-1} \mathrm{dia}^{-1}$ ) no respectivo tratamento foi obtida pelo somatório dos valores obtidos nas pesagens, dividido pelo número de pesagens.

Com base na fibra em detergente neutro indigerível (FDNi) das amostras (dieta total, sobras e fezes), foi determinada a digestibilidade da dieta, segundo Silva \& Queiroz (2002).

A excreção fecal em $\mathrm{kg} \mathrm{d}^{-1}$ de MS foi calculada utilizando-se a fórmula:

$E F=(F D N i C \div \% F D N i F) \times 100$, em que: $\mathrm{FDNiC}(\mathrm{kg}$ $\left.\mathrm{d}^{-1}\right)=$ FDNi consumida; e FDNiF $(\%)=$ FDNi fecal.

A digestibilidade da matéria seca consumida (DMS), correspondente a cada tratamento, foi determinada pela a seguinte expressão:

$D M S(\%)=100-(\% F D N i C M S \div \% F D N i F) \times 100$, em que, \%FDNiCMS = teor de FDNi na MS consumida, sendo obtida da seguinte forma:

$\%$ FDNiCMS $=($ FDNi em $\mathrm{kg} \div$ CMS em $\mathrm{kg}) \times 100$.

A digestibilidade aparente da MS, MO, PB e FDN foi determinada utilizando-se a fórmula: $D(\%)=100-$ [(\%FDNiCMS $\div \%$ FDNiF) X $(\% N F \div \% N C M S) \times 100]$, em que:

Tabela 2. Composição químico-bromatológica do capim-elefante (\%MS) submetido às adubações química (CEQ) e orgânica (CEO)

\begin{tabular}{|c|c|c|c|c|c|c|c|c|c|c|c|}
\hline & \multirow{2}{*}{ MS -\% } & \multicolumn{10}{|c|}{$\%$ da MS } \\
\hline & & MO & PB & FDN & $\mathbf{E L L}^{1}$ & NDT $^{2}$ & $\mathbf{C a}$ & $\mathbf{P}$ & $\mathrm{Na}$ & $\mathbf{K}$ & Mg \\
\hline CEQ & 24,33 & 93,93 & 6,76 & 74,46 & 0,91 & 42,04 & 0,29 & 0,12 & 0,06 & 1,99 & 0,21 \\
\hline CEO & 19,59 & 92,29 & 8,21 & 71,52 & 0,99 & 45,17 & 0,29 & 0,17 & 0,06 & 2,36 & 0,19 \\
\hline Média & 21,96 & 93,11 & 7,49 & 72,99 & 0,95 & 43,61 & 0,29 & 0,14 & 0,06 & 2,17 & 0,20 \\
\hline
\end{tabular}

${ }^{1}$ Calculado com base na equação ELL (Mcal/kgMS) $=2,863-0,0262$ (\%FDN) (Mertens, 1987) e expresso da mesma maneira. ${ }^{2}$ Calculado com base na equação \%NDT $=(\mathrm{ELL}+0,12) / 0,0245(\mathrm{NRC}, 2001)$

Rev. Ceres, Viçosa, vv. 58, n.4, p. 452-461, jul/ago, 2011 
$\% \mathrm{NF}=$ teor do nutriente nas fezes; e $\% \mathrm{NCMS}=$ teor do nutriente na MS.

A estimativa da produção de proteína bruta microbiana (PBM) foi efetuada com base na equação proposta pelo NRC (2001), em que:

$P B M\left(\mathrm{~kg} \mathrm{~d}^{-1}\right)=6,25(-31,86+26,12 \times 1,02 \times \mathrm{MOD})$, em que $\operatorname{MOD}\left(\mathrm{kg} \mathrm{d}^{-1}\right)=$ matéria orgânica digerida.

A determinação da energia líquida total (ELT) do capim-elefante na dieta total foi calculada a partir da energia líquida para produção de leite $\left(\mathrm{EL}_{\mathrm{L}}\right)$ e da energia líquida para mantença $\left(\mathrm{EL}_{\mathrm{m}}\right)$, com base nas informações do NRC (2001). O peso vivo (PV) das vacas assumido para cálculo da $\mathrm{EL}_{\mathrm{m}}$ foi a média de pesos do início e final do período. A energia líquida para mantença foi calculada de acordo com a equação proposta pelo NRC (2001):

$$
E L_{m}=0,08 \text { Mcal } x \mathrm{~kg}^{0,75}
$$

A energia líquida para produção de leite foi calculada segundo a fórmula proposta pelo NRC (2001):

$$
E L_{L}=(0,3512+0,0962 \times \% g) \times P L, \text { em que: }
$$

\% $\mathrm{g}=$ teor de gordura no leite; e PL $(\mathrm{kg})=$ produção de leite.

O procedimento usado para calcular a EL do capimelefante (ELV), expressa em Mcal kg-1 MS, foi: que:

$$
E L V=\left\{\left[\left(E L_{L}+E L_{m}\right) \div C M S\right]-\left(P_{c} \times E L C\right)\right\} \div P_{v}, \mathrm{em}
$$

$\mathrm{P}_{\mathrm{v}}=$ equivalente proporcional do volumoso incluído na dieta total $(0,4 ; 0,5 ;$ e 0,6$) ; \mathrm{P}_{\mathrm{c}}=$ equivalente proporcional do concentrado incluído na dieta total $(0,4 ; 0,5$; e 0,6$)$; e ELC $($ Mcal / kg CMS $)=$ EL no concentrado.

Para obtenção da ELC, foram utilizados valores tabelados, citados pelo NRC (2001).

Para obtenção da EL na dieta total (ELDT em Mcal/ $\mathrm{kgMS}$ ) foi empregada a seguinte fórmula:

$$
E L D T=\left(P_{c} x E L C\right)+\left(P_{v} x E L V\right)
$$

Para determinação do NDT, utilizou-se a equação proposta pelo NRC (2001), a partir da ELV:

$$
\% N D T=(E L V+0,12) \div 0,0245
$$

O pH ruminal foi estimado utilizando a equação proposta por Allen (1997).

$p H=3,98+(0,011 \times F D N)+(0,040 \times M O C)+(0,031 \times$ $F D N F)+(0,18 \times$ PL12) $-(0,15 \times$ PL13),

em que: MOC $\left(\mathrm{kg} \mathrm{dia}^{-1}\right)=$ matéria orgânica consumida; $\mathrm{FDNF}=\mathrm{FDN}$ da forragem $(\%$ na MS); e PLI2 e 3 = comprimento indexado da partícula cortada acima de $0,3 \mathrm{~cm}$ $(0,0519)$ e longa $(-0,2145)$.
Os dados foram submetidos a análises de variância e regressão, e as médias dos fatores qualitativos foram comparadas utilizando-se o teste $\mathrm{F}$ a $5 \%$ de probabilidade. Os modelos foram escolhidos com base na significância dos coeficientes de regressão, utilizando-se o teste t, a 5\% de probabilidade, e no coeficiente de determinação. Para todas as análises estatísticas foi utilizado, o Sistema de Análises Estatística e Genética - SAEG(SAEG, 2007).

\section{RESULTADOS E DISCUSSÃO}

Houve incremento dos constituintes $(\mathrm{p}<0,05)$ das médias contidas na Tabela 3 quando se aumentaram os níveis de concentrado, exceto para a FDN. O NDT calculado aumentou em 6,30 e 5,93\% ao passar do nível de 400 para $500 \mathrm{~g}$ e deste para o de $600 \mathrm{~g}$, respectivamente. A FDN do capim-elefante, calculada com base nas Tabelas 2 e 3, supriu 83,86; 79,24; e 74,16\% de toda a FDN nos níveis de 400, 500 e $600 \mathrm{~g}$ de concentrado, respectivamente. No nível de 600 g, a FDN do capim-elefante atingiu o limiar mínimo de $75 \%$ sugerido pelo NRC (2001), em que a quantidade de FDN oriunda da forragem deve conter no mínimo de $25 \%$ do teor total de FDN da dieta e não deve ser inferior a $75 \%$.

A adubação do capim-elefante com esterco de curral possibilitou formular dietas com teores maiores $(\mathrm{P}<0,05)$ de $\mathrm{PB}$ e $\mathrm{P}$, menores $(\mathrm{P}<0,05)$ em FDN e similares $(\mathrm{P}>0,05)$ em NDT e Ca (Tabela 3).

Segundo Mertens (1987), o benefício aditivo de 2,57\% a mais de NDT e 3,36\% a menos de FDN é um aspecto nutricional relevante ao CMS, advindo das dietas que incluem CAO. É sabido também, que a fibra exerce efeito significativo sobre a capacidade do animal em consumir matéria seca. Contudo, os 9,34\% a mais de água nessa dieta podem ter anulado tal benefício em melhorar o consumo (Robison et al.,1990).

Os consumos apresentaram médias diferentes $(\mathrm{P}<0,05)$ em cada nível de concentrado (Tabela 4). O CMS aumentou em 12,9 $\mathrm{g} \mathrm{dia}^{-1}$ para cada $\mathrm{g}$ de concentrado acrescido à dieta, em contraste com a queda de 7,0 $\mathrm{g} \mathrm{dia}^{-1}$ no consumo de capim-elefante.

Segundo Mertens (1987), o consumo é limitado em níveis de retículo-rúmen (fill ou enchimento ruminal), principalmente pela FDN da dieta, e fisiológico quando a dieta supre as necessidades energéticas do animal. $\mathrm{O}$ aumento de 4,23\% (Tabela 4) no CMS da dieta do nível de 500 para 600 g pode estar relacionado à alta densidade energética e às frações elevadas da FDN de alimentos não-fibrosos. Em adição, ao se observar a exigência nutricional desses animais (NRC, 2001), verifica-se que a dieta com nível de $600 \mathrm{~g}$ de concentrado pode estar além das exigências nutricionais, considerando que já se encontravam no terço médio da lactação.

Rev. Ceres, Viçosa, v. 58, n.4, p. 452-461, jul/ago, 2011 
Tabela 3. Teores médios de MS, FDN, PB, NDT, Ca e P da dieta total oferecida com diferentes níveis de concentrado associado ao capim-elefante submetido às adubações química e orgânica

\begin{tabular}{|c|c|c|c|c|c|c|c|}
\hline \multirow[b]{2}{*}{ Constituintes } & \multicolumn{7}{|c|}{ Concentrado (\% MSDT) } \\
\hline & $\begin{array}{c}400 \\
\mathrm{~A}\end{array}$ & $\begin{array}{c}(\mathbf{B}-\mathbf{A}) / \mathbf{A} \\
\%\end{array}$ & $\begin{array}{c}500 \\
\text { B }\end{array}$ & $\begin{array}{c}(\mathrm{C}-\mathrm{B}) / \mathrm{B} \\
\%\end{array}$ & $\begin{array}{c}600 \\
C\end{array}$ & Regressão & $\mathbf{r}^{2}$ \\
\hline $\mathrm{MS}^{1}$ & 29,84 & $+10,54$ & 32,99 & $+9,29$ & 36,05 & $\hat{\mathrm{Y}}=174,3472+0,3105^{*} \mathrm{X}$ & 0,99 \\
\hline $\mathrm{FDN}^{2}$ & 52,23 & $-11,81$ & 46,06 & $-14,52$ & 39,37 & $\hat{\mathrm{Y}}=780,3125-0,6429 * * \mathrm{X}$ & 0,99 \\
\hline $\mathrm{PB}^{2}$ & 10,89 & $+10,15$ & 11,99 & $+11,88$ & 13,42 & $\hat{\mathrm{Y}}=57,7750+0,1265 * * \mathrm{X}$ & 0,99 \\
\hline $\operatorname{NDT}^{2,3}$ & 58,42 & $+6,30$ & 62,09 & $+5,93$ & 65,78 & $\hat{\mathrm{Y}}=437,0125+0,368 * * \mathrm{X}$ & 0,99 \\
\hline $\mathrm{Ca}^{2}$ & 0,42 & $+7,62$ & 0,45 & $+7,08$ & 0,48 & $\hat{\mathrm{Y}}=2,9356+0,003166^{* *} \mathrm{X}$ & 0,99 \\
\hline \multirow[t]{3}{*}{$\mathrm{P}^{2}$} & 0,27 & $+11,68$ & 0,31 & $+11,11$ & 0,34 & $\hat{\mathrm{Y}}=1,4436+0,003258 * * \mathrm{X}$ & 0,99 \\
\hline & \multicolumn{7}{|c|}{ Adubações } \\
\hline & $\begin{array}{c}\text { Química } \\
Q(C)\end{array}$ & & & Orgânica $\mathbf{O}$ & & $\begin{array}{l}(\mathrm{O}-\mathrm{Q}) / \mathrm{Q} \\
\%(\%)\end{array}$ & \\
\hline $\mathrm{MS}^{1}$ & $35,95^{\mathrm{A}}$ & & & $29,96^{\mathrm{B}}$ & & $-17,64$ & \\
\hline $\mathrm{FDN}^{2} 2$ & $46,67^{\mathrm{A}}$ & & & $45,10^{\mathrm{B}}$ & & $-3,36$ & \\
\hline $\mathrm{PB}^{2}$ & $11,71^{\mathrm{B}}$ & & & $12,49^{\mathrm{A}}$ & & $+6,63$ & \\
\hline $\mathrm{NDT}^{2,3}$ & $61,31^{\mathrm{A}}$ & & & $62,88^{\mathrm{A}}$ & & $+2,57$ & \\
\hline $\mathrm{Ca}^{2}$ & $0,45^{\mathrm{A}}$ & & & $0,45^{\mathrm{A}}$ & & $-0,44$ & \\
\hline $\mathrm{P}^{2}$ & $0,29^{\mathrm{B}}$ & & & $0,32^{\mathrm{A}}$ & & $+7,43$ & \\
\hline
\end{tabular}

${ }^{1} \mathrm{Em} \mathrm{g} \mathrm{kg}^{-1}$ de matéria natural. ${ }^{2}$ Em \% de matéria seca.

${ }^{3}$ Com base na Tabela 2, nos níveis e no teor de NDT do concentrado $=80,40 \%$ (NRC, 2001). Médias subscritas na mesma linha diferem $(\mathrm{P}<0,05)$ pelo teste $\mathrm{F} . *=\mathrm{P}<0,05 \mathrm{e}^{* *}=\mathrm{P}<0,01$ pelo teste $\mathrm{t}$.

Tabela 4. Consumos médios de MS e MO da dieta total (MSDT), do volumoso (MSV) e do concentrado (MSC) em dietas com diferentes níveis de concentrado, associado ao capim-elefante submetido às adubações química e orgânica

\begin{tabular}{|c|c|c|c|c|c|c|c|}
\hline \multirow{3}{*}{ Itens } & \multicolumn{7}{|c|}{ Nível de concentrado (g/kg MSDT) Concentrate levels (g/kg DMTD) } \\
\hline & 400 & $(\mathbf{B}-\mathbf{A}) / \mathbf{A}$ & 500 & $(\mathrm{C}-\mathrm{B}) / \mathrm{B}$ & 600 & & \\
\hline & $\mathbf{A}$ & $\%$ & B & $\%$ & $\mathbf{C}$ & Regressão & $\mathbf{r}^{2}$ \\
\hline \multicolumn{8}{|c|}{ Consumo (kg /dia) } \\
\hline MS & 13,41 & $+14,39^{1}$ & 15,34 & $+4,23$ & 15,99 & $\hat{\mathrm{Y}}=8,4629+0,0129 * * \mathrm{X}$ & 0,92 \\
\hline MSV & 8,05 & $-4,72$ & 7,67 & $-13,30$ & 6,65 & $\hat{\mathrm{Y}}=10,9309-0,007 * * \mathrm{X}$ & 0,93 \\
\hline MSC & 5,37 & $+42,83$ & 7,67 & $+21,77$ & 9,34 & $\hat{Y}=-2,4747+0,02 * * X$ & 0,99 \\
\hline \multirow[t]{2}{*}{ MO } & 12,80 & $+14,61$ & 14,67 & $+4,57$ & 15,34 & $\hat{\mathrm{Y}}=7,9078+0,0127 * \mathrm{X}$ & 0,93 \\
\hline & \multicolumn{7}{|c|}{ Consumos (\% PV) } \\
\hline MS & 2,65 & $(+13,51)$ & 3,01 & $(+2,99)$ & 3,1 & $\hat{\mathrm{Y}}=1,8019+0,0022 * \mathrm{X}$ & 0,89 \\
\hline MSV & 1,59 & $(-5,66)$ & 1,50 & $(-14,00)$ & 1,29 & $\hat{\mathrm{Y}}=2,2119-0,0015^{* *} \mathrm{X}$ & 0,94 \\
\hline MSC & 1,06 & $(+41,50)$ & 1,50 & $(+20,66)$ & 1,81 & $\hat{\mathrm{Y}}=-0,4163+0,0037 * * \mathrm{X}$ & 0,98 \\
\hline \multirow[t]{4}{*}{ MO } & 2,53 & $(+13,44)$ & 2,87 & $(+3,48)$ & 2,97 & $\hat{Y}=1,6878+0,0022 * X$ & 0,90 \\
\hline & \multicolumn{7}{|c|}{ Adubações } \\
\hline & \multicolumn{3}{|c|}{ Química (Q) } & \multicolumn{2}{|c|}{ Orgânica (O) } & \multicolumn{2}{|l|}{$(\mathrm{O}-\mathrm{Q}) / \mathrm{Q}(\%)$} \\
\hline & \multicolumn{5}{|c|}{ Consumo (kg /dia) } & & \\
\hline MS & & $15,30^{\mathrm{A}}$ & & 14,53 & & $-5,03$ & \\
\hline MSV & & $7,76^{\mathrm{A}}$ & & $7,16^{\mathrm{A}}$ & & $-7,73$ & \\
\hline MSC & & $7,54^{\mathrm{A}}$ & & $7,37^{\mathrm{A}}$ & & $-2,25$ & \\
\hline \multirow[t]{2}{*}{ MO } & & $14,73^{\mathrm{A}}$ & & 13,81 & & $-6,25$ & \\
\hline & \multicolumn{5}{|c|}{ Consumo (\% PV) } & & \\
\hline MS & & $2,97^{\mathrm{A}}$ & & $2,87^{\mathrm{A}}$ & & $-3,37$ & \\
\hline MSV & & $1,51^{\mathrm{A}}$ & & $1,41^{\mathrm{A}}$ & & $-6,62$ & \\
\hline MSC & & $1,46^{\mathrm{A}}$ & & $1,45^{\mathrm{A}}$ & & $-0,68$ & \\
\hline MO & & $2,86^{\mathrm{A}}$ & & $2,72^{\mathrm{A}}$ & & $-4,90$ & \\
\hline
\end{tabular}

Médias subscritas na mesma linha diferem $(\mathrm{P}<0,05)$ pelo teste $\mathrm{F}$.

$*=\mathrm{P}<0,05 \mathrm{e}^{* *}=\mathrm{P}<0,01$ pelo teste $\mathrm{t}$.

Rev. Ceres, Viçosa, vv. 58, n.4, p. 452-461, jul/ago, 2011 
A utilização de CAO na preparação de dieta total não diferiu significativamente do CAQ $(\mathrm{P}>0,05)$ em todos os parâmetros de consumo observados na Tabela 4. Numericamente houve diminuição de CMS com a utilização da CAO.

O consumo de matéria seca e de nutrientes de uma dieta para vacas leiteiras é muito dependente do status fisiológico da vaca (Hutjens, 1995) e da composição em FDN da dieta (Okine et al., 1997).

O consumo médio de FDN foi similar $(\mathrm{P}>0,05)$ nos diferentes níveis de concentrado (Tabela 5). Entretanto, do nível 500 para 600 g o consumo de FDN reduziu 12,45\%; em contrapartida, ocorreu diminuição de $0,99 \%$ do nível de 400 para 500 g de concentrado. Já os consumos de PB, $\mathrm{Ca} \mathrm{e} \mathrm{P}$ foram maiores $(\mathrm{P}<0,05)$ à medida que aumentou $\mathrm{O}$ nível de concentrado.

O maior consumo de $\mathrm{PB}, \mathrm{Ca}$ e $\mathrm{P}$ entre os níveis de 400 e $500 \mathrm{~g}$ de concentrado deve-se ao maior CMS da dieta total e do concentrado, como pode ser visto na Tabela 4.

O desbalanceamento de nutrientes de uma dieta pode ser um fator inibidor do consumo de matéria seca e de nutrientes dessa dieta, devido ao aumento excessivo de metabólitos (Illius \& Jessop, 1996). No nível de 600 g, o consumo de FDN em \% PV apresentou valor dentro dos parâmetros de variação relatado por Mertens (1997). Abaixo de $600 \mathrm{~g}$, os animais apresentaram maiores consumos de FDN, chegando a 1,39\% do PV no nível de 400 g. O CMS entre os extremos de níveis de concentrado variou em uma amplitude de 19,24\% (Tabela 4), apresentando consumo médio de FDN de $6,75 \mathrm{~kg} \mathrm{~d}^{-1}$. Soares et al. (2001ab), Lopes et al. (2004) e Sousa et al. (2008) verificaram amplitudes de consumo de FDN para vacas leiteiras similares ao encontrado neste trabalho, onde obtiveram 1,1 a $2,7 \%$ FDN/PV.

Os valores obtidos para digestibilidade da MS, MO e $\mathrm{PB}$ foram similares $(\mathrm{P}>0,05)$ nos diferentes níveis de concentrado. A digestão e a fração digerida por dia da FDN diminuíram $(\mathrm{P}<0,05)$ e das frações de MO e PB aumentaram $(\mathrm{P}<0,05)$ em níveis crescentes de concentrado na dieta total (Tabela 6).

Ao passar do nível de 400 para 600 g de concentrado, ocorreu decréscimo de $14,01 \%$ na digestão e $31,21 \%$ na fração digerida por dia da FDN, sendo maior a redução observada entre os níveis de 400 e 500 g. Esses resultados são justificados pelos obtidos em Nocek (1997), Mertens (1997) e NRC (2001).

O aumento no consumo de concentrado entre os níveis de 400 e $500 \mathrm{~g}$ (Tabela 4) possibilitou a maior fração digerida por dia de $\mathrm{PB}$.

Os valores de digestibilidade de CAO foram maiores $(\mathrm{P}<0,05)$ para a MSDT, MO e PB e similares $(\mathrm{P}>0,05)$ para FDN e as frações digeridas diariamente de MO, FDN e PB (Tabela 6). Reis (2000) relatou que o capim-elefante cultivado com esterco de curral, em relação ao cultivado com adubação química, apresentou atributos nutricionais de composição químico-bromatológica superiores e teor de FDN inferior, implicando em maior digestibilidade da MSDT, MO e PB. O consumo de 5,30\% a mais de MS do CAQ (Tabela 4), em contraste com seus piores atributos nutricionais e menor $(\mathrm{P}<0,05)$ digestibilidade da FDN

Tabela 5. Consumo médio de FDN, PB, Ca e P na dieta total com diferentes níveis de concentrado, associado ao capim-elefante submetido às adubações química e orgânica

\begin{tabular}{|c|c|c|c|c|c|c|c|}
\hline \multirow[b]{2}{*}{ Itens } & \multicolumn{7}{|c|}{ Concentrado (g /kg MSDT) } \\
\hline & 400 & (B-A)/A & 500 & $(\mathrm{C}-\mathrm{B}) / \mathrm{B}$ & 600 & Regressão & $\mathbf{r}^{2}$ \\
\hline & \multicolumn{7}{|c|}{ Consumo (kg /dia) } \\
\hline FDN & 7,00 & $+0,99^{1}$ & 7,07 & $-12,45$ & 6,19 & $\hat{\mathrm{Y}}=6,75$ & - \\
\hline $\mathrm{PB}$ & 1,47 & $+32,65$ & 1,95 & $+11,28$ & 2,17 & $\hat{\mathrm{Y}}=0,1250+0,0035^{* * X}$ & 0,95 \\
\hline $\mathrm{Ca}$ & 0,0562 & $+23,31$ & 0,0693 & $+11,54$ & 0,0773 & $\hat{\mathrm{Y}}=0,01494+0,000105^{*} * \mathrm{X}$ & 0,98 \\
\hline $\mathrm{P}$ & 0,0368 & $+27,72$ & 0,0470 & $+15,74$ & 0,0544 & $\hat{\mathrm{Y}}=0,002059+0,000087 * * \mathrm{X}$ & 0,99 \\
\hline \multirow[t]{4}{*}{ FDN $(\% \mathrm{PV})$} & 1,38 & $+0,72$ & 1,39 & $-12,95$ & 1,21 & $\widehat{\mathrm{Y}}=1,33$ & - \\
\hline & \multicolumn{7}{|c|}{ Adubações } \\
\hline & \multicolumn{3}{|c|}{ Química (Q) } & \multicolumn{2}{|c|}{ Orgânica (O) } & \multirow[t]{2}{*}{$(\mathrm{O}-\mathrm{Q}) / \mathrm{Q}(\%)$} & \\
\hline & \multicolumn{5}{|c|}{ Consumo (kg /dia) } & & \\
\hline FDN & \multicolumn{2}{|c|}{$7,09^{\mathrm{A}}$} & & \multicolumn{2}{|c|}{$6,42^{\mathrm{B}}$} & \multicolumn{2}{|l|}{$-9,45$} \\
\hline PB & \multicolumn{2}{|c|}{$1,85^{\mathrm{A}}$} & & \multicolumn{2}{|c|}{$1,87^{\mathrm{A}}$} & \multicolumn{2}{|l|}{$+1,08$} \\
\hline $\mathrm{Ca}$ & \multicolumn{2}{|c|}{$0,0694^{\mathrm{A}}$} & & \multicolumn{2}{|c|}{$0,0658^{\mathrm{A}}$} & \multicolumn{2}{|l|}{$-5,19$} \\
\hline $\mathrm{P}$ & \multicolumn{2}{|c|}{$0,0455^{\mathrm{A}}$} & & \multicolumn{2}{|c|}{$0,0466^{\mathrm{A}}$} & \multicolumn{2}{|l|}{$+2,42$} \\
\hline FDN $(\% \mathrm{PV})$ & \multicolumn{2}{|c|}{$1,38^{\mathrm{A}}$} & & \multicolumn{2}{|c|}{$1,28^{\mathrm{B}}$} & $-7,25$ & \\
\hline
\end{tabular}

Médias subscritas na mesma linha diferem $(\mathrm{P}<0,05)$ pelo teste $\mathrm{F}$.

$* *=\mathrm{P}<0,01$ pelo teste $\mathrm{t}$. 
(Tabela 6), não alterou as quantidades de frações digeridas diariamente de MO, FDN e PB do CAQ ou CAO.

Na Tabela 7 estão contidos os valores de PB e NDT, dos quais foram estimadas distintamente as eficiências de síntese de proteína bruta microbiana (PBM) em relação ao NDT e à PB consumida.

Os valores de correlação nos três níveis de concentrado foram para PB consumida, PBM e PBD de 0,33 (P < 0,18), 0,18 ( $\mathrm{P}<0,49)$ e 0,07 $(\mathrm{P}<0,79)$, respectivamente, para o NDT, 0,19 (P < 0,45); e para eficiência de síntese de PBM da PB consumida e do NDT, 0,49 (P<0,04) e 0,09 (P $<0,71)$, respectivamente.

A dieta utilizada na alimentação das vacas deste trabalho não considerou o fracionamento da energia e da proteína, que deve ter sido a principal causa da baixa significância entre aquelas variáveis, exceto para eficiência de utilização de PB consumida, que foi suprida em quantidades adequadas na dieta utilizada.

Tabela 6. Valores médios para digestão da MS, MO, FDN e PB e para a fração digerida de MO, FDN e PB em dietas com diferentes níveis de concentrado, associados ao capim-elefante submetido às adubações química e orgânica

\begin{tabular}{|c|c|c|c|c|c|c|c|}
\hline \multirow[b]{2}{*}{ Itens } & \multicolumn{7}{|c|}{ Concentrado (g/kg MSDT) } \\
\hline & $\begin{array}{c}400 \\
\mathrm{~A}\end{array}$ & $\begin{array}{c}(\mathbf{B}-\mathbf{A}) / \mathbf{A} \\
\%\end{array}$ & $\begin{array}{c}500 \\
\text { B }\end{array}$ & $\begin{array}{c}(\mathbf{C}-\mathrm{B}) / \mathrm{B} \\
\%\end{array}$ & $\begin{array}{c}600 \\
C\end{array}$ & Regressão & $\mathbf{r}^{2}$ \\
\hline \multicolumn{8}{|c|}{ Digestão (\%) } \\
\hline MS & 54,72 & $+4,51^{1}$ & 57,19 & $+3,19$ & 59,02 & $\hat{\mathrm{Y}}=569,76$ & - \\
\hline MO & 56,07 & $+3,76$ & 58,18 & $+3,90$ & 60,46 & $\hat{Y}=582,41$ & - \\
\hline FDN & 43,59 & $-7,90$ & 40,15 & $-6,63$ & 37,48 & $\hat{Y}=556,8305-0,305^{\#} X$ & 0,99 \\
\hline PB & 55,64 & $+11,29$ & 61,92 & $-4,63$ & 59,05 & $\hat{Y}=588,73$ & - \\
\hline \multicolumn{8}{|c|}{ Fração digerida (kg/dia) } \\
\hline MO $(O M)$ & 7,22 & $+13,99$ & 8,23 & $+11,54$ & 9,18 & $\hat{\mathrm{Y}}=3,3183+0,0098 * * \mathrm{X}$ & 0,99 \\
\hline FDN $(N D F)$ & 3,14 & $-12,74$ & 2,74 & $-1,17$ & 2,16 & $\hat{Y}=5,143-0,0049 * * X$ & 0,98 \\
\hline $\mathrm{PB}(C P)$ & 0,86 & $+39,53$ & 1,20 & $+8,33$ & 1,30 & $\hat{\mathrm{Y}}=(42,79+2,152 * * \mathrm{X}) \div 10^{3}$ & 0,90 \\
\hline \multicolumn{8}{|c|}{ Adubações } \\
\hline & \multicolumn{2}{|c|}{ Química (Q) } & \multicolumn{3}{|c|}{ Orgânica $(\mathrm{O})$} & \multirow[t]{2}{*}{$(\mathrm{O}-\mathrm{Q}) / \mathrm{Q}(\%)$} & \\
\hline & \multicolumn{5}{|c|}{ Digestão (\%) } & & \\
\hline MS & \multicolumn{2}{|c|}{$547,13^{\text {в }}$} & \multicolumn{3}{|c|}{$592,38^{A}$} & \multicolumn{2}{|l|}{$+8,27$} \\
\hline MO & \multicolumn{2}{|c|}{$559,40^{\text {В }}$} & \multicolumn{3}{|c|}{$605,43^{A}$} & \multicolumn{2}{|l|}{$+8,23$} \\
\hline FDN & \multicolumn{2}{|c|}{$382,79^{\mathrm{A}}$} & \multicolumn{3}{|c|}{$425,46^{\mathrm{A}}$} & \multicolumn{2}{|l|}{$+11,15$} \\
\hline \multirow[t]{2}{*}{ PB } & & $31^{\mathrm{B}}$ & & $626,14^{\mathrm{A}}$ & & \multirow[t]{2}{*}{$+13,57$} & \\
\hline & \multicolumn{5}{|c|}{ Fração digerida (kg/dia) } & & \\
\hline MO & & $13^{\mathrm{A}}$ & & $8,29^{\mathrm{A}}$ & & $+1,97$ & \\
\hline FDN $(N D F)$ & & $68^{\mathrm{A}}$ & & $2,68^{\mathrm{A}}$ & & 0 & \\
\hline $\mathrm{PB}$ & & $6^{\mathrm{A}}$ & & $1,18^{\mathrm{A}}$ & & $+11,32$ & \\
\hline
\end{tabular}

Médias subscritas na mesma linha diferem $(\mathrm{P}<0,05)$ pelo teste $\mathrm{F}$.

${ }^{\#}=\mathrm{P}<0,1, *=\mathrm{P}<0,05 \mathrm{e}^{* *}=\mathrm{P}<0,01$ pelo teste $\mathrm{t}$.

Tabela 7. Médias de PB, NDT e eficiência de síntese microbiana (EM) de PBM em dietas com diferentes níveis de concentrado, associado ao capim-elefante submetido às adubações química e orgânica

\begin{tabular}{|c|c|c|c|c|c|c|c|}
\hline \multirow[b]{2}{*}{ Item } & \multicolumn{4}{|c|}{ Concentrado ( $\left.\mathrm{g} \mathrm{kg}^{-1} \mathrm{MSDT}\right)$} & \multicolumn{3}{|c|}{ Adubações } \\
\hline & 400 & 500 & 600 & Média & Química (Q) & Orgânica $(\mathbf{O})$ & $(O-Q) / Q$ \\
\hline & \multicolumn{6}{|c|}{ Parâmetros (kg /dia) } & $\%$ \\
\hline PBC & 1,57 & 1,95 & 2,18 & 1,90 & 1,93 & 1,88 & $-2,64$ \\
\hline $\mathrm{PBM}^{1}$ & 1,05 & 1,23 & 1,39 & 1,22 & 1,20 & 1,25 & $+3,85$ \\
\hline PBD & 0,86 & 1,20 & 1,29 & 1,12 & 1,06 & 1,18 & $+11,51$ \\
\hline $\mathrm{NDT}^{2}$ & 7,37 & 8,39 & 9,36 & 8,37 & 8,29 & 8,45 & $+1,97$ \\
\hline EM (gPBM/kgPBD) & 668,85 & 630,30 & 636,39 & 645,18 & 622,73 & 664,27 & $+6,67$ \\
\hline EM $(\mathrm{gPBM} / \mathrm{kgNDT})$ & 142,65 & 146,79 & 148,50 & 145,98 & 144,86 & 147,53 & $+1,84$ \\
\hline
\end{tabular}

PBD e PBC = Valores de proteína bruta digerida e consumida apresentados na Tabela 6.

${ }^{1}$ Com base no modelo matemático proposto pelo NRC (2001), em função da MOD observada. ${ }^{2}$ Com base na fórmula NDT (kg/dia) = $1,02 *$ MOD proposta NRC (2001).

Rev. Ceres, Viçosa, vv. 58, n.4, p. 452-461, jul/ago, 2011 
Nos parâmetros deste trabalho a PB consumida foi sempre maior que a PBD em nível ruminal, indicando que parte da PB foi perdida em forma de ureia e excretada via urinária e, ou, parte não sofreu a degradação pela microbiota ruminal. A fração verdadeiramente utilizada de PB pela microbiota ruminal foi aquela degradada no rúmen, a qual, em comparação com a PBM estimada, apresentou coeficiente de correlação igual a $0,76(\mathrm{P}<0,003)$. Com base neste valor, é possível que considerável parte do nitrogênio $(\mathrm{N})$ da fração da PBD tenha sido incorporada na microbiota ruminal e, dessa forma, poupou-se energia que deveria ser gasta pelo fígado para neutralizar o escape da amônia do ecossistema ruminal. A fração de $\mathrm{N}$ da dieta não-utilizada pela microbiota ruminal, seja aquela perdida via urinária ou como proteína verdadeira, pode ser uma das causas do baixo coeficiente de correlação observado para eficiência de síntese de PBM da PB consumida.

Satter \& Slyter (1974) relataram que, em dietas com níveis de até $13 \%$ de PB e adequado suprimento de energia, a produção microbiana ruminal atinge a estabilidade. No nível de $600 \mathrm{~g}$ de concentrado, o teor de PB esteve acima de $13 \%$ (Tabela 4), sendo, portanto, possível que nesse nível, em relação aos outros, a reciclagem de $\mathrm{N}$ tenha sido menor em favor de maior excreção de $\mathrm{N}$-ureia via urina e, ou, leite.

Na dieta com nível de $400 \mathrm{~g}$ de concentrado, a eficiência de síntese de PBM da PB consumida foi 5,61\% superior à média nos outros dois níveis de concentrado (500 e $600 \mathrm{~g}$ ), denotando maior reciclagem de N.
O ARFC (1993) e o NRC (2001) sugeriram que a proporção ideal de produção microbiana ruminal seja da ordem de $130 \mathrm{~g}$ de PBM kg-1 de NDT e $11 \mathrm{~g} \mathrm{PBM} / \mathrm{MJ}$ de energia metabolizável (EM). Com base nesses trabalhos e nos dados observados na Tabela 8, pode-se verificar que a eficiência de utilização do NDT, na média dos três níveis de concentrado, foi $12,29 \%$ superior, quando comparada com o NRC (2001), e 28,01\% inferior, quando comparada com o AFRC (1993).

Os valores médios de produção e composição química do leite não variaram $(\mathrm{P}>0,05)$ nos diferentes níveis de concentrado (Tabela 8). Contudo, entre os níveis de 400 e $500 \mathrm{~g}$ houve melhoria percentual maior que entre os níveis de 500 e $600 \mathrm{~g}$. Este incremento entre os níveis de 400 e $500 \mathrm{~g}$ se deve ao maior consumo de MS e concentrado (Tabela 4) e dos nutrientes PB, Ca e P, em contraste com o consumo de FDN, que permaneceu estável (Tabela 5). Em adição, a fração digerida da $\mathrm{PB}$ como também da $\mathrm{MO}$, em relação à de FDN, foi maior (Tabela 6).

Com base nas Tabelas 3 e 8, observou-se que, quando o nível de concentrado passou de 400 para 500 g e de 400 para 600 g, a produção de leite teve acréscimo de 9,27 e $11,12 \%$ e do NDT de 6,30 e $12,60 \%$, respectivamente. O aumento de $6 \%$ do NDT não contribuiu para elevar a produção de leite, porém melhorou a condição corporal dos animais, tendo o seu peso vivo aumentado em $+2,80 \%$, visto que eles eram mestiços.

A dieta preparada com CAO não apresentou diferença (P $>0,05)$ nas médias de produção e composição química do

Tabela 8. Produção média de leite, gordura, proteína e sólidos totais (ST) do leite em vacas recebendo diferentes níveis de concentrado, associado ao capim-elefante submetido às adubações química e orgânica

\begin{tabular}{|c|c|c|c|c|c|c|c|}
\hline \multirow[t]{3}{*}{ Produção } & \multicolumn{5}{|c|}{ Concentrado (g/kg MSDT) } & \multirow{3}{*}{ Regressão } & \multirow{3}{*}{$\mathbf{r}^{2}$} \\
\hline & 400 & $(\mathbf{B}-\mathbf{A}) / \mathbf{A}$ & 500 & $(\mathrm{C}-\mathrm{B}) / \mathrm{B}$ & 600 & & \\
\hline & $\mathbf{A}$ & $\%$ & B & $\%$ & C & & \\
\hline \multicolumn{8}{|c|}{ Produção de leite (kg/vaca/dia) } \\
\hline Real & 13,49 & $+9,27$ & 14,74 & $+1,70$ & 14,99 & $\hat{\mathrm{Y}}=14,41$ & - \\
\hline $4 \%$ de gordura & 13,96 & $+10,46$ & 15,42 & $+0,78$ & 15,54 & $\hat{\mathrm{Y}}=14,97$ & - \\
\hline 305 dias de lactação ${ }^{1}$ & 11,74 & $+9,45$ & 12,85 & $+1,56$ & 13,05 & $\hat{Y}=12,55$ & - \\
\hline Gordura ( $\mathrm{g} / \mathrm{kg}$ de leite) & 42,57 & $+3,19$ & 43,93 & $-3,00$ & 42,61 & $\hat{Y}=43,04$ & - \\
\hline Proteína (g/kg de leite) & 32,57 & $+2,98$ & 33,54 & $+2,15$ & 34,26 & $\hat{\mathrm{Y}}=33,46$ & - \\
\hline \multirow[t]{4}{*}{$\mathrm{ST}(\mathrm{g} / \mathrm{kg}$ de leite $)$} & 135,14 & $+1,72$ & 137,46 & $+0,49$ & 136,79 & $\hat{Y}=136,46$ & - \\
\hline & \multicolumn{7}{|c|}{ Adubações } \\
\hline & \multicolumn{3}{|c|}{ Química (Q) } & \multicolumn{2}{|c|}{ Orgânica (O) } & $(\mathbf{O}-\mathbf{Q}) / \mathrm{Q}(\%)$ & \\
\hline & \multicolumn{5}{|c|}{ Produção de leite (kg/ vaca/ dia) } & & \\
\hline Real & \multicolumn{3}{|c|}{$14,77^{\mathrm{A}}$} & \multicolumn{2}{|c|}{$14,04^{\mathrm{A}}$} & $-4,94$ & \\
\hline $4 \%$ de gordura & \multicolumn{3}{|c|}{$15,38^{\mathrm{A}}$} & \multicolumn{2}{|c|}{$14,57^{\mathrm{A}}$} & $-5,27$ & \\
\hline 305 dias de lactação & \multicolumn{3}{|c|}{$12,86^{\mathrm{A}}$} & \multicolumn{2}{|c|}{$12,24^{\mathrm{A}}$} & $-4,82$ & \\
\hline Gordura ( $\mathrm{g} / \mathrm{kg}$ de leite) & \multicolumn{3}{|c|}{$43,22^{\mathrm{A}}$} & \multicolumn{2}{|c|}{$42,84^{\mathrm{A}}$} & $-0,88$ & \\
\hline Proteína (g/ kg de leite) & \multicolumn{3}{|c|}{$34,04^{\mathrm{A}}$} & \multicolumn{2}{|c|}{$32,87^{\text {в }}$} & $-3,44$ & \\
\hline ST (g/ kg de leite) & \multicolumn{3}{|c|}{$137,07^{\mathrm{A}}$} & \multicolumn{2}{|c|}{$135,86^{\mathrm{A}}$} & $-1,21$ & \\
\hline
\end{tabular}

Médias subscritas na mesma linha diferem $(\mathrm{P}<0,05)$ pelo teste $\mathrm{F}$. 
leite, exceto para proteína, que foi menor $(\mathrm{P}<0,05)$ nas dietas com CAO (Tabela 8). Adiferença relativa de 4,94\% na produção de leite, observada na Tabela 8, em dietas contendo CAO, é o reflexo da queda no CMS em consequência do alto teor de água constatado nas dietas com CAO.

Os valores de energia líquida para lactação (ELL) e do NDT do capim-elefante foram decrescentes $(\mathrm{P}<0,05)$ com a elevação dos níveis de concentrado na dieta total (Tabela 9). A redução na digestibilidade e na fração digerida da FDN, observada na Tabela 6, contribuiu para o fato observado na Tabela 9. Shriver et al. (1986), trabalhando com dietas de alto concentrado, verificaram que o aumento no fluxo da dieta elevou a eficiência microbiana, ao contrário do $\mathrm{pH}$, e não encontraram nenhum efeito sobre digestão de carboidratos estruturais.

Do nível de 400 para 600 g de concentrado ocorreu decréscimo da ELL e do NDT do capim-elefante da ordem de 37,50 e 33,41\%, respectivamente. Já nos trabalhos de Galyean \& Goetsch (1993), foi observado decréscimo a partir de $300 \mathrm{~g}$ concentrado, possivelmente devido ao menor teor de lignina na FDN da forragem utilizada.

O decréscimo verificado da ELL de 0,3675 Mcal kg-1 MS de capim-elefante entre os níveis de 400 e 600 g pode refletir, de acordo com o potencial biológico do animal, na produção de leite, e ou, no ganho de peso. Esta observação não considera os cálculos de balanceamento de dietas para bovinos leiteiros em que a energia é determinada a partir do somatório da fração ofertada por cada ingrediente, sem se importar com a influência da variação do nível dos ingredientes da dieta no ecossistema ruminal, especialmente no $\mathrm{pH}$ e na taxa de fluxo da digesta, reduzindo a digestão das frações com taxa de digestão lenta e, por conseguinte, a eficiência microbiana.

Contrastando os valores de ELV e NDT do capim-elefante no nível de $400 \mathrm{~g}$ de concentrado com os mesmos valores referentes à média do capim-elefante nos dois sistemas de adubação (Tabela 2), notou-se que não houve oscilação significante, o que indicou que a inclusão de concentrado à dieta básica de capim-elefante até o nível de 400 g não deve ter reduzido a digestibilidade desse capim.

Com base no estimador proposto por Allen (1997), os valores de $\mathrm{pH}$ ruminal decresceram de 6,47 para 6,01 com a elevação do nível de concentrado na dieta, indicando interferências na digestão do capim-elefante, as quais já foram relatadas anteriormente com base nas informações de Shriver et al. (1986).

Tabela 9. Valores médios de energia líquida total para lactação (ELL-Mcal/kg MS) e NDT (\% MS) do capim-elefante submetido às adubações química e orgânica e misturado em diferentes níveis de concentrado

\begin{tabular}{|c|c|c|c|c|c|c|c|}
\hline \multirow{3}{*}{ Itens } & \multicolumn{7}{|c|}{ Concentrado ( $\%$ MSDT)) } \\
\hline & 400 & $(\mathbf{B}-\mathbf{A}) / \mathbf{A}$ & 500 & $(\mathrm{C}-\mathrm{B}) / \mathrm{B}$ & 600 & Regressão & $\mathbf{r}^{2}$ \\
\hline & $\mathbf{A}$ & $\%$ & B & $\%$ & C & & \\
\hline \multirow{4}{*}{$\begin{array}{l}\text { ELL } \\
\text { NDT }^{1}\end{array}$} & 0,98 & $-16,98$ & 0,81 & $-24,72$ & 0,61 & $\hat{\mathrm{Y}}=1,7179-0,00183 * * \mathrm{X}$ & 0,82 \\
\hline & 44,89 & $-15,12$ & 38,11 & $-21,54$ & 29,90 & $\hat{\mathrm{Y}}=750,1883-0,7469 * * \mathrm{X}$ & 0,82 \\
\hline & & \multicolumn{6}{|c|}{ Adubações } \\
\hline & & \multicolumn{3}{|c|}{ Química (Q) } & \multicolumn{2}{|c|}{ Orgânica (O) } & \\
\hline$\overline{\text { ELL }}$ & & & \multicolumn{2}{|c|}{$0,8136^{\mathrm{A}}$} & \multicolumn{2}{|c|}{$0,8173^{\mathrm{A}}$} & \\
\hline $\mathrm{NDT}^{1}$ & & & $381,05^{\mathrm{A}}$ & & \multicolumn{2}{|c|}{$382,59^{\mathrm{A}}$} & \\
\hline
\end{tabular}

${ }^{1}$ Estimador $(\% \mathrm{NDT}=(\mathrm{ELL}+0,12) / 0,0245(\mathrm{NRC}, 2001)$. Médias subscritas na mesma linha diferem $(\mathrm{P}<0,05)$ pelo teste $\mathrm{F} . * *=\mathrm{P}<0,01$ pelo teste $\mathrm{t}$.

\section{CONCLUSÕES}

A dieta total contendo $400 \mathrm{~g}$ de concentrado por kg de MS possibilitou produção de leite com melhor eficiência e a mesma composição química em comparação com níveis de concentrado superiores.

As dietas balanceadas com nível acima de $400 \mathrm{~g}$ de concentrado subestimaram a contribuição da energia oriunda do volumoso no cômputo total, por reduzir a digestão da fibra.

Vacas leiteiras recebendo dieta total com volumoso de capim-elefante submetido à adubação orgânica ou química não apresentaram diferença na produção e composição química do leite.

\section{REFERÊNCIAS}

Agricultural and Food Research Council-AFRC (2003) Energy and protein requirements of ruminants. Cambridge, Cambridge University Press. 159p.

Allen MS (1997) Relationship between fermentation acid production in the rumen and the requirement for physically effective fiber. Journal of Dairy Science, 80:1447-1462.

Coelho DT \& Rocha JAA (1981) Práticas de processamento de produtos de origem animal. Viçosa, Imprensa Universitária/UFV. 58p.

Galyean ML \& Goetsch AL (1993) Utilization of forage fiber by ruminants. In: Jung HG \& Buxton RD (Eds). Forage cell wall structure and digestibility. Madison, ASA-CSSA-SSSA. p.34-62.

Hutjens MF (1995) Feeding applications for the high producing cow. In: Cornell Nutrition Conference For Feed Manufactures, Ithaca. Proceedings, Cornell University. p.34-41. 
Illius AW \& Jessop NS (1996) Metabolic constraints on voluntary intake in ruminants. Journal of Animal Science, 74:3052-3062.

Lopes FCF, Aroeira LJM \& Rodriguez NM (2004) Efeito da suplementação e do intervalo de pastejo sobre a qualidade da forragem e consume voluntário de vacas Holandês x Zebu em lactação em pastagem de capim-elefante. Arquivo Brasileiro de Medicina Veterinária e Zootecnia, 56:355-362.

Mertens DR (1987) Predicting intake and digestibility using mathematical models of ruminal function. Journal of Animal Science, 64:1548-1558.

Mertens DR (1994) Regulation of forage intake. In: Fohey JR GC (Ed). Forage quality, evaluation, and utilization. Madison, ASACSSA-SSSA. p.450-532.

Mertens DR (1997) Creating a system for meeting the fiber requirements of Dairy cows. Journal of Dairy Science, 80:14631481 .

Mora PJG (1995) Utilização de diferentes níveis de grão de soja (Glycine $\max$ L.) cru moído em rações concentradas e determinação da energia líquida da silagem de milho (Zea mays L.) para vacas em lactação. Dissertação de Mestrado. Universidade Federal de Viçosa, Viçosa. 104p.

Nocek JE (1997) Bovine acidosis: implications on laminitis. Journa of Dairy Science, 80:1005-1028.

National Research Council-NRC (2001) Nutrient requirements of beef cattle. 7 ed. Washington, DC, 242p.

Oliveira MA, Reis RB, Ladeira MM, Pereira IG, Franco GL, Saturnino HM, Coelho SG, Artunduaga MAT, Faria BN \& Souza Júnior JA (2007) Produção e composição do leite de vacas alimentadas com dietas com diferentes proporções de forragem e teores de lipídeos. Arquivo Brasileiro de Medicina Veterinária e Zootecnia, 59:759-766.

Okine EK, Khorasani GR \& Kennely JJ (1997) Effects of source of forage and level of concentrate on chewing activity and milk production response in late lactation cows. Canadian Journal of Animal Science, 77:253-258.

Reis CS (2000) Utilização do Capim-Elefante (Pennisetum purpureum Schum. Cv. Mineiro), Submetido à adubação Química e Orgânica, na Alimentação de Vacas Leiteiras. Dissertação de Mestrado. Universidade Federal de Viçosa, Viçosa. 108p.
Robinson PH, Burgess PL \& McQueen RE (1990) Influence of moisture content of mixed rations on feed intake and milk production of dairy cows. Journal of Dairy Science, 73:2916-2921.

SAEG: (2007) Sistema para Análises Estatísticas. Versão 9.1. Viçosa, UFV.

Satter LD \& Slyter LL (1974) Effect of ammonia concentration on rumen microbial protein production in vitro. British Journal Nutrition, 32:199-208.

Shriver BJ, Hoover WH \& Sargent RJ (1986) Fermentation of a high concentrate diet as affected by ruminal $\mathrm{pH}$ and digesta flow. Journal of Dairy Science, 69:413-418.

Silva DJ \& Queiroz AC (2002) Analise de alimentos: Métodos químicos e biológicos. $3^{\mathrm{a}}$ ed. Viçosa, UFV. $235 \mathrm{p}$.

Soares JPG, Salman AKD \& Berchielli TT (2001a) Predição do consumo voluntário do capim-tanzânia (Panicum maximum, J. cv. Tanzânia), sob pastejo, por vacas em lactação, a partir das características de degradação. Revista Brasileira de Zootecnia, 30:2176-2182.

Soares JPG, Aroeira LJM \& Verneque RS (2001b) Estimativa do consumo e da taxa de passagem do capim-elefante (Pennisetum purpureum Schum.) sob pastejo de vacas em lactação. Revista Brasileira de Zootecnia, 30:2183-2191.

Souza BM, Saturnino HM \& Borges ALCC (2008) Estimativa de consumo de matéria seca e de fibra em detergente neutro por vacas leiteiras sob pastejo, suplementadas com diferentes quantidades de alimento concentrado. Arquivo Brasileiro de Medicina Veterinária e Zootecnia, 60:890-895.

Van Soest PJ (1994) Nutritional ecology of the ruminant. 2.ed. Cornell, Cornell University Press. 476p.

Van Soest PJ, Robertson JB \& Lewis BA (1991) Methods for dietary fiber, neutral detergent fiber, and nonstarch polysaccharides in relation to animal nutrition. Journal of Dairy Science, 74:3583-3597

Vilela D, Alvim MJ \& Campos OF (1996) Produção de leite de vacas holandesas em confinamento ou em pastagem de coast-cross. Revista da Sociedade Brasileira de Zootecnia, 25:1228-1244.

Wattiaux MA (1994) Energy and protein metabolism. In Technical dairy guide: nutrition and feeding. Chapter 3 . Wisconsin, Madison. p.33-43. 